\title{
Prostate Cancer by Whitmore-Jewett
}

\section{Stage}

National Cancer Institute

\section{Source}

National Cancer Institute. Prostate Cancer by Whitmore-Jewett Stage. NCI Thesaurus. Code C7079.

A term that refers to the staging of prostate carcinoma according to the WhitmoreJewett staging system. 